\title{
CONTRIBUTION A L'ÉTUDE POLAROGRAPHIQUE ET BACTÉRIOLOGIQUE DU LAIT PASTEURISÉ A HAUTE TEMPÉRATURE
}

\author{
par \\ V. GHEORGHE et V. MANU \\ Vétérinaires \\ Institut d'hygiène — Centrale laitière \\ Bucarest
}

Les transformations subies par les protéines du lait sous l'influence de certains agents physico-chimiques peuvent être mises en évidence par l'analyse polarographique. En utilisant comme support la solution cobalteuse de Brdicka [1], on obtient une double vague caractéristique pour les protéines, dont la hauteur et la position varient en fonction de l'agent physico-chimique et de l'intensité de son action. L.-M. Buruiana et V. Pavlu $[2,3,4]$ ont utilisé cette méthode afin d'étudier les protéines du lait et l'effet de certains agents physico-chimiques sur celles-ci. Peu d'ouvrages seulement ont analysé, du point de vue polarographique, les transformations des protéines du lait sous l'influence de la chaleur $[2,5,6]$.

Compte tenu de la nécessité de détruire, par la pasteurisation, un grand nombre de germes saprophytes, les germes pathogènes étant certainement tués aux températures officielles de pasteurisation [7] (quoique divers auteurs aient estimé que certaines souches de bacilles de la tuberculose soient susceptibles dans des conditions particulières, de résister à un chauffage à $85^{\circ}$ pendant 5-10 minutes [8]), nous avons considéré théoriquement que la transformation des protéines du lait pourrait avoir lieu en même temps que celle des protéines des cellules bactériennes et que, à partir d'un certain degré, la dégradation protéique du lait aurait done comme corollaire la destruction d'un nombre assez grand de bactéries. La vérification expérimentale de ces considérations constituerait le fondement de la technique de pasteurisation du lait à haute température en vue d'établir les meilleurs barèmes de température et de temps.

A cet effet nous avons appliqué la méthode polarographique et effectué la détermination du nombre total des germes.

En même temps, nous avons étudié l'aspect alimentaire diététique de la pasteurisation du lait à haute température par l'appréciation la plus précise du degré de la dénaturation subie par les protéines du lait. 


\section{Matériel et méthodes de travail}

Les études ont porté sur des échantillons de lait de vache de grande collecte, dont la teneur en matière grasse était de 3 p. 100 4,2 p. 100 et l'acidité de $16^{\circ}-18^{\circ} \mathrm{T}$. Les déterminations sont effectuées sur le sérum du lait non-dégraissé. On n’a pas procédé au dégraissage par centrifugation parce que, ainsi que nous l'avons constaté dans nos essais préliminaires, il n'y a pas de différence remarquable, entre les vagues du lait entier et celles du lait dégraissé, qui puisse influencer les résultats (la hauteur des vagues du lait dégraissé est un peu plus réduite que la hauteur du lait nondégraissé) [9]. La caséine a été éliminée par précipitation avec $1,7 \mathrm{ml}$ d'acide acétique $1 \mathrm{~N}$ pour $10 \mathrm{ml}$ de lait. Afin de séparer le sérum du précipité de la caséine, on a utilisé la filtration sur double papier filtre. A l'aide d'une micropipette, on a prélevé du filtrat, une quantité rigoureusement exacte de 0,02 ml (quantité établie par tâtonnement) qu'on a introduite dans la cellule d'électrolyse, garnie au préalable d'une quantité adéquate de mercure, comme anode. Ensuite, on a ajouté la solution-support de eobalt $(0,01 \mathrm{M}$ $\mathrm{CoCl}_{2}-0,1 \mathrm{~N} \mathrm{NH_{3 }}$ et $0,1 \mathrm{~N} \mathrm{NH}_{4} \mathrm{Cl}$ ) et l'on a effectué la polarographie.

Les déterminations polarographiques ont été réalisées à l'aide d'un polarographe du type Heyrovsky LP 55, dont les cellules d'électrolyse en verre comportent une électrode de platine. La durée d'égouttage à la cathode était de 3 secondes. La température pendant les déterminations a été de $25^{\circ} \mathrm{C}$. On a employé des séries de 5 à 6 échantillons représentant un même lot de lait pasteurisé à diverses températures, en inscrivant le même nombre de polarogrammes sur le papier polarographique photosensible. La polarographie a eu lieu entre les limites de tension de $-1,2 \mathrm{~V}$ à $-1,9 \mathrm{~V}$, en utilisant un courant continu de $3,8 \mathrm{~V}$. On a établi, par tâtonnements, une sensibilité de travail de $1 / 100$ et c'est par rapport à ce chiffre qu'on a tracé la plupart des polarogrammes de l'étude. Il a été aussi adopté, pour nos derniers polarogrammes; la sensibilité de 1/70 qui, en présentant des vagues plus hautes, permet d'obtenir une précision plus grande encore pour la détermination de la hauteur des vagues polarographiques.

\section{Résultat}

Les premières études expérimentales d'orientation ont été effectuées dans les conditions du laboratoire, par le chauffage du lait à diverses températures de $70^{\circ}$ à $85^{\circ} \mathrm{C}$, dans des éprouvettes aux parois minces, immergées dans un bain d'eau-thermostat, porté à la température désirée. L'augmentation de la température du lait contenu dans l'éprouvette à partir de $30^{\circ} \mathrm{C}$ jusqu'à la température de pasteurisation demandait environ 2 minutes. Après la pasteurisation, qui durait 30 secondes, le lait était immédiate- 
ment refroidi pendant 5 secondes environ, par immersion du tube dans un courant d'eau du robinet.

Les expériences avec des échantillons de lait pasteurisé à $70^{\circ}, 75^{\circ}$, et $80^{\circ} \mathrm{C}$ ont montré que la hauteur des vagues polarographiques, obtenues à ces températures, était très proche ou égale à la hauteur des vagues présentées par le même échantillon du lait non-soumis au traitement thermique (lait cru). Donc, à ces températures ainsi qu'à la durée de 30 secondes, il ne se produit pas des transformations dans les albumines du lait qui puissent être détectées par polarographie ou bien elles ont peu d'importance et deviennent plus intenses vers $80^{\circ} \mathrm{C}$.

Etant donné le but poursuivi, on a effectué ensuite des expériences seulement aux températures de $80^{\circ}, 83^{\circ}$ et $85^{\circ} \mathrm{C}$ et on a constaté que, par comparaison avec du lait cru de la même provenance, considéré comme étalon, la hauteur des vagues polarographiques obtenues à $80^{\circ}$ était très proche de celle du lait cru. La hauteur des vagues à $83^{\circ} \mathrm{C}$ était sensiblement plus réduite. Une diminution plus aceentuée de la hauteur a été remarquée pour les vagues polarographiques obtenues par la pasteurisation à $85^{\circ} \mathrm{C}$, ceci pour tous les échantillons analysés.

Afin de pouvoir étudier les différences entre la hauteur des vagues polarographiques à diverses températures, on a considéré le rapport mathématique entre la hauteur des vagues obtenues pour le lait eru et la hauteur des vagues pour chacune des températures, rapportées au même échantillon de lait. On a obtenu ainsi des valeurs comparables pour les échantillons de laits de diverses provenances.

Dans la deuxième étape des travaux expérimentaux, le lait a été pasteurisé, en couche mince, dans des installations industrielles à plaques, étant chauffé à $80^{\circ}, 83^{\circ}, 85^{\circ}$ et $90^{\circ} \mathrm{C}$, pendant 16 secondes. Les résultats obtenus pour 8 lots de lait sont présentés dans le tableau I (pour chacun des lots, on a analysé en parallèle trois échantillons). Les données du tableau viennent confirmer les observations résultant de l'étude des échantillons de lait pasteurisé dans les conditions de laboratoire. On peut ainsi constater que, pour chaque lot de lait analysé, la hauteur des vagues polarographiques du lait pasteurisé décroît à mesure que la température de chauffage est plus élevée. On confirme la constatation que la décroissance des vagues polarographiques, exprimée par le rapport entre les valeurs du lait cru et du lait pasteurisé, est plus nette de $83^{\circ}$ à $85^{\circ}$, étant de deux fois (la première vague protéique) jusqu'à quatre fois (la deuxième vague protéique) plus accentuée que dans les limites de température de $80^{\circ}-83^{\circ}$ et $85^{\circ}-90^{\circ}$. La hauteur de la deuxième vague fait mieux ressortir cette différenciation.

Afin de vérifier la reproductibilité de ce phénomène on a répété les polarogrammes pour les mêmes échantillons de lait et dans les mêmes conditions de travail, après une heure, puis après deux 
TABLEAU I

VARIATION DES HAUTEURS DES VAGUES POLAROGRAPHIQUES EN FONCTION DE LA TEMPÉRATURE DE PASTEURISATION DU LAIT, PENDANT 16 SECONDES (MOYENNE DES 24 POLAROGRAMMES POUR CHAQUE TEMPÉRATURE DE PASTEURISATION)

\begin{tabular}{|c|c|c|c|c|}
\hline \multirow{3}{*}{ Lait pasteurisé à } & \multicolumn{4}{|c|}{$\begin{array}{l}\text { Rapport entre la hauteur de la vague du lait cru } \\
\text { et celle de la vague du lait pasteurisé }\end{array}$} \\
\hline & \multicolumn{2}{|c|}{$\begin{array}{l}\text { Première vague } \\
\text { protéique }\end{array}$} & \multicolumn{2}{|c|}{$\begin{array}{l}\text { Deuxième vague } \\
\text { protéique }\end{array}$} \\
\hline & $\begin{array}{c}\text { Valeur } \\
\text { moyenne } \\
\text { arithmétique }\end{array}$ & $\begin{array}{l}\text { Différence } \\
\text { entre les } \\
\text { valeurs } \\
\text { moyennes } \\
\text { arithmétiques }\end{array}$ & $\begin{array}{c}\text { Valeur } \\
\text { moyenne } \\
\text { arithmétique }\end{array}$ & $\begin{array}{c}\text { Différence } \\
\text { entre les } \\
\text { valeurs } \\
\text { moyennes } \\
\text { arithmétiques }\end{array}$ \\
\hline $80^{\circ} \mathrm{C}$ & 1,10 & & 1,08 & \\
\hline $83^{\circ} \mathrm{C}$ & 1,20 & $\begin{array}{l}\text { de } 80^{\circ} \text { à } 83^{\circ} \\
+\quad 8 \% \\
\end{array}$ & 1,16 & $\begin{array}{c}\text { de } 80^{\circ} \text { à } 83^{\circ} \\
+\quad 7 \% \\
\end{array}$ \\
\hline $85^{\circ} \mathrm{C}$ & 1,43 & $\begin{array}{l}\text { de } 83^{\circ} \text { à } 85^{\circ} \\
+19 \% \\
\end{array}$ & 1,48 & $\begin{aligned} & \text { de } 83^{\circ} \text { à } 85^{\circ} \\
&+27 \% \\
&\end{aligned}$ \\
\hline $90^{\circ} \mathrm{C}$ & 1,51 & $\begin{array}{l}\text { de } 85^{\circ} \text { à } 90^{\circ} \\
\quad+\quad 5 \%\end{array}$ & 1,60 & $\begin{array}{c}\text { de } 85^{\circ} \text { à } 90^{\circ} \\
+\quad 8 \%\end{array}$ \\
\hline
\end{tabular}

et trois jours de conservation (les échantillons étant conservés à 4-5 $5^{\circ}$ ). On a constaté des différences infimes de la hauteur des vagues, à partir de 0,5 jusqu'à $1 \mathrm{~mm}$, d'un jour à l'autre, par comparaison avec le premier polarogramme, lesquelles différences sont associées aux erreurs admissibles de l'analyse polarographique, ainsi qu'à certaines transformations physico-chimiques intervenues pendant la conservation des échantillons ; toutefois ces différences ne modifient pas le tableau général des échantillons de lait du premier jour. Au $4^{\mathrm{e}}$ et $5^{\mathrm{e}}$ jour de conservation, la hauteur des vagues ne présente plus les variations antérieurement constatées, mais des déviations remarquables d'un échantillon à l'autre.

Afin d'étudier les caractéristiques polarographiques du lait bouilli, on a effectué, dans les mêmes conditions de travail, la polarographie d'échantillons de lait cru et du même lait chauffé en tube à essai pendant 10 minutes dans un récipient contenant de l'eau bouillante. On a obtenu un polarogramme tout à fait différent par comparaison non seulement au lait cru, mais encore au lait 
pasteurisé à diverses températures de $72^{\circ}$ à $90^{\circ}$. Dans le cas du lait bouilli, les vagues polarographiques ont complètement disparu, présentant une courbe en plateau, semblable à celle obtenue par la polarographie de l'épreuve témoin (avec, seulement, la solutionsupport de chlorure de cobalt).

TABLEAU II

VARIATION DU NOMBRE TOTAL DES GERMES ET DES BACTÉRIES COLIFORMES EN FONCTION DE LA TEMPÉRATURE DE PASTEURISATION DU LAIT, PENDANT 16 SECONDES (MOYENNE ARITHMÉTIQUE DES 15 ÉCHANTILLONS)

\begin{tabular}{lcccccc}
$\begin{array}{c}\text { Températures de } \\
\text { pasteurisation }\end{array}$ & $70^{\circ} \mathrm{C}$ & $72^{\circ} \mathrm{C}$ & $75^{\circ} \mathrm{C}$ & $80^{\circ} \mathrm{C}$ & $83^{\circ} \mathrm{C}$ & $85^{\circ} \mathrm{C}$ \\
\hline $\begin{array}{l}\text { Nombre total des } \\
\text { germes/ml lait }\end{array}$ & 44160 & 31690 & 16400 & 9650 & 4257 & 458 \\
\hline Pourcentage & 100 & 71 & 37 & 21 & 9 & 1 \\
\hline $\begin{array}{c}\text { moyenne } \\
\text { pour } 3 \\
\text { Bactéries coliformes/ml }\end{array}$ & $\begin{array}{c}\text { échantillons } \\
16\end{array}$ & 0 & 0 & 0 & 0 & 0 \\
\hline \hline
\end{tabular}

Pour constater en quelle mesure le comportement polarographique des protéines du lait pasteurisé à diverses températures correspond à la variation du nombre total des bactéries du lait, on a analysé 15 lots de lait pasteurisé expérimentalement dans les mêmes installations industrielles, aux températures de $70^{\circ}, 72^{\circ}$, $75^{\circ}, 80^{\circ}, 83^{\circ}$ et $85^{\circ} \mathrm{C}$, pendant 16 secondes. Les résultats moyens arithmétiques (tableau II) indiquent la décroissance du nombre des bactéries parallèlement à l'augmentation de la température de pasteurisation. En général la réduction est de moitié pour une augmentation de température de 3 à $5^{\circ}$. On a remarqué une diminution brusque du nombre total des germes pour une pasteurisation à $85^{\circ} \mathrm{C}$; la population survivante n'est respectivement que le dixième et le centième du nombre des germes persistant après pasteurisation à $83^{\circ}$ et $70^{\circ} \mathrm{C}$. Les bactéries coliformes présentes dans trois des 15 lots pasteurisés à $70^{\circ}$ (9-250 et 110 germes/ ml) n'ont plus été trouvées aux autres températures de pasteurisation.

\section{Discussion}

La décroissance de la hauteur des vagues polarographiques, notamment celle de la deuxième vague, parallèlement à l'augmentation de la température de chauffage du lait, a été signalée aussi 
par d'autres auteurs $[2,5]$. Ce phénomène est associé au degré de dénaturation, par coagulation, de l'albumine du lait. A mesure que la température augmente, la quantité d'albumine coagulée s'accroît. Ainsi qu'il a été constaté [10], la lactalbumine est coagulée dans les limites de température allant de $68,5^{\circ}$ à $88^{\circ} \mathrm{C}$.

$\mathrm{Au}$ commencement de ce siècle même, Orla Jensen [11] a démontré que, par le chauffage du lait cru pendant 5 minutes aux températures variant de $70^{\circ}$ à $140^{\circ}$, l'albumine non-coagulée, trouvée dans le liquide filtré après précipitation à l'acide acétique, décroissait à partir de 13,13 p. 100 dans le sérum du lait cru, jusqu'à 1,59 p. 100 dans le sérum du lait chauffé à $80^{\circ}$, A $90^{\circ}$ et aux températures plus hautes, on ne trouvait plus d'albumine non-coagulée dans le sérum. Par comparaison, il ressort de nos observations que, par chauffage du lait de $72^{\circ}$ à $80^{\circ} \mathrm{C}$ pendant 16 et 30 secondes, la dénaturation du lait par coagulation, est très peu importante. Elle est plus accentuée à $83^{\circ}$ et très intense à $85^{\circ}$. Au-dessus de $85^{\circ}$, par exemple à $90^{\circ}$, l'analyse polarographique indique une réactivité plus faible du sérum à cause, certainement, de la coagulation d'une quantité assez grande d'albumine. Au cas du chauffage à $100^{\circ}$, pendant 10 minutes, les vagues polarographiques sont complètement effacées, le polarogramme présentant la forme d'un plateau, ce qui signifie qu'à cette température, toute la quantité d'albumine est coagulée.

Le saut dans le degré de dénaturation de l'albumine à la tem. pérature de pasteurisation du lait à $85^{\circ} \mathrm{C}$ correspond à un degré considérable de destruction de la flore bactérienne saprophyte. Cette coïncidence peut être attribuée à la dénaturation, par coagulation, de l'albumine contenue dans les cellules bactériennes, ce qui entraîne leur destruction massive.

Ces résultats pourraient fournir des données scientifiques en vue d'adopter une des méthodes de pasteurisation du lait à haute température, lesquelles méthodes ne sont pas encore bien établies jusqu'à présent. On applique sur une grande échelle, les méthodes H.T.S.T. (High Temperature Short Time), c'est-à-dire 15 secondes à $72^{\circ}$, et également la pasteurisation à des températures plus hautes encore, de $80^{\circ}$ à $90^{\circ}$ (Flash). Chacun de ces procédés a ses adeptes résolus.

Si l'on tend vers l'adoption d'une méthode où la dénaturation des protéines soit extrêmement réduite, tout en assurant la destruction des germes pathogènes, et une réduction de la flore bactérienne saprophyte assurant une conservabilité raisonnable, il résulte que la pasteurisation dans les limites de $72^{\circ}$ à $80^{\circ}$ est la plus indiquée. Les températures intermédiaires de pasteurisation, adoptées pour certaines installations industrielles, ne sont pas justifiées parce que les résultats sont à peu près les mêmes dans ces limites de température. En ce cas, il est à préférer la pasteurisation à $72^{\circ}$, au moins pour des considérations d'ordre technique concernant l'économie d'énergie calorique utilisée, ainsi que pour des raisons 
diététiques concernant la certitude d'avoir obtenu du lait nondénaturé. Il y a toutefois le risque d'un décroissement accidentel de la température de pasteurisation, à laquelle les germes pathogènes pourraient résister.

La pasteurisation à $83^{\circ}$ a comme résultat un certain degré de dénaturation de l'albumine du lait et une réduction considérable du nombre des germes saprophytes (d'environ 1/10), par comparaison à la pasteurisation à $72^{\circ}$. C'est une température de pasteurisation dont on doit tenir compte dans la pratique de l'industrie du lait, qui assure la conservabilité du lait pour une période plus longue et qui se trouve à la limite d'une dénaturation brusque des protéines produite par les températures un peu plus hautes.

La pasteurisation à $85^{\circ}$ présente un caractère différent. Une coagulation brusque de l'albumine correspond à une réduction considérable du nombre des germes saprophytes (moyenne de 500 germes survivants au $\mathrm{ml}$ ), laquelle réduction a été également signalée par J. Pien [12], dans les limites des températures de pasteurisation de $84^{\circ}$ à $86^{\circ}$. Cette température peut représenter la limite supérieure des températures de pasteurisation. Au-dessus de $85^{\circ}$, les albumines, qui ont été coagulées pour la plupart, commencent à être dénaturées par modification plus accentuée de leur structure chimique. Cette modification pourrait seulement être évitée par la réduction de la durée de pasteurisation jusqu'à une seconde, ce qui est extrêmement difficile à réaliser dans la pratique. En outre la réduction du nombre total des germes au-dessous de $500 \mathrm{au} \mathrm{ml}$ n'a plus de raison pratique; elle est d'ailleurs difficile à effectuer à cause de la thermorésistance des bactéries à $85^{\circ}$. Au-dessus de cette température on ne peut concevoir que la stérilisation, qui implique toutefois une technologie différente en ce qui concerne la conservation du lait.

\section{Résumé}

En comparant les polarogrammes présentés par le sérum du lait pasteurisé dans le laboratoire ainsi que dans des conditions industrielles expérimentales, aux températures de $70^{\circ}, 75^{\circ}, 80^{\circ}, 83^{\circ}$, $85^{\circ}$ et $90^{\circ} \mathrm{C}$, pendant $16-30$ secondes, on a constaté, à $85^{\circ}$, un décroissement brusque dans la hauteur de la double vague polarographique, caractéristique des protéines, par rapport à la hauteur des vagues du lait pasteurisé à $83^{\circ} \mathrm{C}$, ce qui indique une transformation plus intense de l'albumine du lait (température critique). A $80^{\circ}$ et au-dessous, on n'a constaté qu'un décroissement peu important de la hauteur des vagues, par comparaison au lait cru. Du point de vue bactériologique, on a observé à la température critique de $85^{\circ}$, une réduction brusque du nombre des germes, comme résultat de la forte transformation subie par l'albumine protoplasmique de la cellule bactérienne, ce qui est similaire aux modifications de l'albumine du lait, constatées par la polarographie. 
En vertu des résultats obtenus, les méthodes de pasteurisation du lait à haute température sont analysées en considération de deux critères principaux : le critère diététique, concernant les transformations de l'albumine, et le critère bactériologique, concernant la destruction des germes. On souligne les avantages pratiques offerts par la pasteurisation à $85^{\circ} \mathrm{C}$.

\section{Summary}

The polarograms shown by the whey of the milk pasteurized in the laboratory as well as under experimental industrial conditions at various temperatures of $70^{\circ}, 75^{\circ}, 80^{\circ}, 83^{\circ}, 85^{\circ}$ and $90^{\circ} \mathrm{C}$ for 16-30 seconds were compared, and a sudden decrease in the height of the double polarographic wave which is specific to proteins was noted as compared to the height of the waves of the milk pasteurized at $83^{\circ} \mathrm{C}$, which proves that milk albumin was more severely modified (critical temperature). The decrease in the height of the waves at and over $80^{\circ}$ was found to be unimportant as compared to raw milk. From the bacteriological point of view, a sudden reduction of the number of germs was noted at the critical temperature of $85^{\circ}$ as a result of the severe alteration in the protoplasmatic albumin of the bacterial cell, which is similar to the changes in milk albumin established by polarography.

On the basis of the results obtained the methods for milk pasteurization at higher temperatures are analysed in consideration of two main criteria, i.e. the dietetical criterion concerning the changes in the albumin, and the bacteriological criterion concerning the total number of germs. The practical advantages of the pasteurization at $85^{\circ} \mathrm{C}$ are emphasized.

\section{BIBLIOGRAPHIE}

[1] Brezina (M.) and Zuman (P.), Polarography in Medicine, Biochemistry and Pharmacy. Interscience Publishers, New York, London, 1958, p. 597.

[2] BuruianA (L. M.) et PAvlu (V.). Le Lait, 1957, XXXVII, Nos 369-370, 601-615.

[3] Buruiana (L. M.) et Pavlu (V.). Le Lait, 1959, XXXIX, 481-488.

[4] Buruiana (L. M.) et Pavlu (V.). Milchwissenschaft, 1963, 18, 12, 613-617.

[5] GAL (T.). Prumysl Potravin, 1954, 5, 206-208.

[6] Nangniot (P.) et Coppens (R.). Bull. Inst. Agron. Gembloux, 1962, 30, 3/4, 314-319. Dairy Sci. Abstrs., 1964, vol. 26, No 12, 1132.

[7] Nevot (A.), LAFONT (Ph). et Lafont (J.). Le Lait, 1959, XXXIX, No $388,488-495$.

[8] Moureau (M.), Bretey (J.) et Roy (D.). Presse médicale, 1960, 68, 261-263.

[9] Kozlov (B.) et Novikova (N.). Molocinaia Prom., 1965, No 4, 27-29.

[10] Koxama (S.) et Suzuki (K.). Bull. Coll. Agric. Vet. Med. Nihon Univ., 1962 (15), 46-53. Dairy Sci. Abstrs., 1964, vol. 26, No 12, 271.

[11] Lindet (L.). Le lait, la crème, le beurre, les fromages, Gauthier-Villars, Paris, 1907 , p. 146.

[12] Piwn (J.). Le Lait, 1960, No 398, 481-511. 